\title{
Invisible Neutrino Decay Could Resolve IceCube's Track and Cascade Tension
}

\author{
Peter B. Denton ${ }^{*}$ and Irene Tamborra ${ }^{\dagger}$ \\ Niels Bohr International Academy and DARK, Niels Bohr Institute, University of Copenhagen, \\ Blegdamsvej 17, 2100, Copenhagen, Denmark
}

(Received 21 May 2018; published 20 September 2018)

\begin{abstract}
The IceCube Neutrino Observatory detects high energy astrophysical neutrinos in two event topologies: tracks and cascades. Since the flavor composition of each event topology differs, tracks and cascades can be used to test the neutrino properties and the mechanisms behind the neutrino production in astrophysical sources. Assuming a conventional model for the neutrino production, the IceCube data sets related to the two channels are in $>3 \sigma$ tension with each other. Invisible neutrino decay with lifetime $\tau / m=10^{2} \mathrm{~s} / \mathrm{eV}$ solves this tension. Noticeably, it leads to an improvement over the standard nondecay scenario of more than $3 \sigma$ while remaining consistent with all other multimessenger observations. In addition, our invisible neutrino decay model predicts a reduction of $59 \%$ in the number of observed $\nu_{\tau}$ events which is consistent with the current observational deficit.
\end{abstract}

DOI: 10.1103/PhysRevLett.121.121802

Introduction.-The IceCube Neutrino Observatory measures high energy astrophysical neutrinos with energies reaching up to few PeVs [1-3]. While numerous source candidates have been proposed to interpret the observed data, no clear picture has yet emerged [4-7].

According to the conventional framework, adopted in this work, high energy astrophysical neutrinos are produced primarily by charged pion decay. Charged pions decay to a muon and a muon neutrino, and the muon in turn decays to a positron, electron neutrino, and a muon antineutrino, resulting in a neutrino flavor ratio at the source of $\nu_{e}: \nu_{\mu}: \nu_{\tau}=1: 2: 0$, each with approximately the same energy. After neutrino oscillations, the flavor ratio at Earth is roughly $1: 1: 1$ leading to the expectation that the spectral distributions of neutrinos will be the same for any flavor, see, e.g., $[4,8]$. This is independent of the source class since any mechanism that produces high energy neutrinos will do so dominantly as a result of charged pion decays. Hence, within this picture, the only possible result is equal fluxes for each flavor.

Single power law (SPL) and broken power law (BPL) fits have been considered to interpret the neutrino data [9-17]. They favor a SPL, with a possible break to explain the excess of events below $100 \mathrm{TeV}[14,18]$.

IceCube is partially sensitive to the flavor state of the neutrino through two distinct event topologies: track events resulting dominantly from $\nu_{\mu}$ interactions [3], and nearly

Published by the American Physical Society under the terms of the Creative Commons Attribution 4.0 International license. Further distribution of this work must maintain attribution to the author(s) and the published article's title, journal citation, and DOI. Funded by SCOAP ${ }^{3}$. spherical cascade events resulting dominantly from $\nu_{e}$ and $\nu_{\tau}$ interactions [19]. The IceCube Collaboration has interpreted each of these data sets in terms of the true per-flavor neutrino flux at Earth under the assumption that the flavor ratio remains constant at $1: 1: 1$ for all energies and that the flux follows a SPL [9]. It is found that those two different channels produce results in tension with each other [3], as shown in Fig. 1.

IceCube finds that the best fit per-flavor astrophysical spectral index and normalization from the track analysis over $E_{\nu} \in[194 \mathrm{TeV}, 7.8 \mathrm{PeV}]$ is $\gamma_{t, \mathrm{IC}}=2.13 \pm 0.13$, $\Phi_{t, \mathrm{IC}}=0.90_{-0.27}^{+0.30}[3]$, and the best fit from the cascade

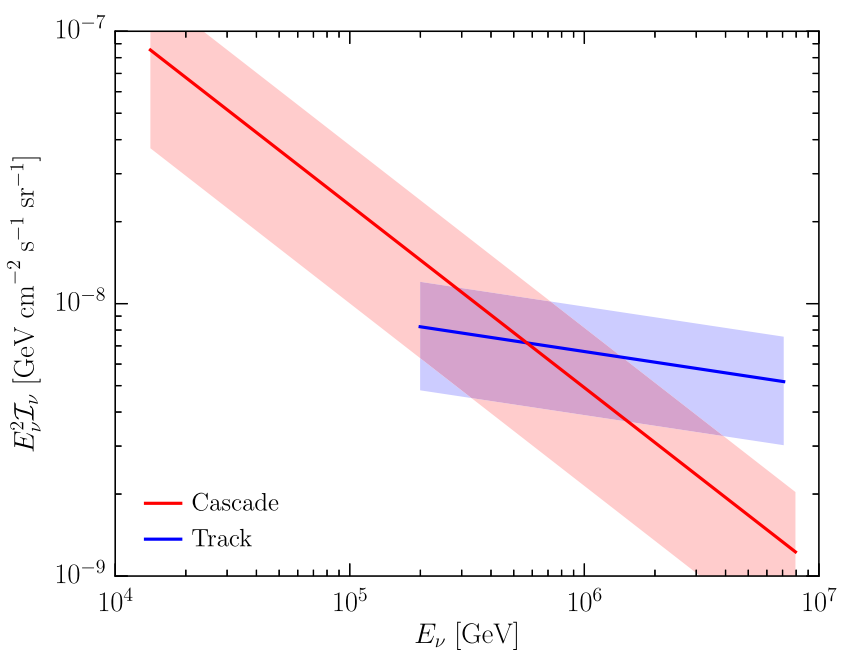

FIG. 1. IceCube track [3] and cascade [19] data samples. The tension between the two data samples is driven on the high energy end by the observation of six tracks with energies $E_{\nu}>1 \mathrm{PeV}$. On the low energy side, there is an apparent excess of events in the cascade channel [18]. 
analysis over $E_{\nu} \in[13 \mathrm{TeV}, 7.9 \mathrm{PeV}]$ is $\gamma_{c, \mathrm{IC}}=2.67_{-0.13}^{+0.12}$, $\Phi_{c, \text { IC }}=2.3_{-0.6}^{+0.7}$ [19], where $\gamma_{i}$ is the spectral index and $\Phi_{i}$ is the flux normalization at $E_{\nu}=100 \mathrm{TeV}$ in units of $10^{-18} \mathrm{GeV}^{-1} \mathrm{~cm}^{-2} \mathrm{sr}^{-1} \mathrm{~s}^{-1}$.

In this Letter, we combine spectral and flavor information simultaneously to investigate the tension between the data sets associated to the two event topologies. We explore several modifications to the standard picture of the high energy astrophysical neutrino flux beyond what is foreseen within the Standard Model [20].

We determine the diffuse intensity at Earth after oscillations, convert this into the per-flavor intensity from each of the track and cascade channels, and fit a power law to each assuming a 1:1:1 flavor ratio to compare a model to IceCube's observations. We then compare the normalizations and spectral indices to the measured ones by combining both tracks and cascades under the assumption that the correlation between the normalizations and spectral indexes are small. Invisible neutrino decay provides a good fit to the data and is preferred over the Standard Model at more than $3 \sigma$, removing the tension. Our proposed solution is not in contradiction with existing multimessenger constraints, and it also explains the current deficit in the observation of $\nu_{\tau}$ events.

Standard neutrino source model.-For the sake of generality, we model the neutrino spectral distribution in such a way to be agnostic about the mechanism of the neutrino production, i.e., $p \gamma$ or $p p$ interactions. We consider a general BPL model at the source, parametrized by the break energy in the source frame $\tilde{E}_{\nu, b}$ and the change in the spectral index $\Delta$, such that the spectral index below the break energy is $\gamma$ and it is $\gamma+\Delta$ above it $[4-7,21]$. The SPL case is then recovered for $\Delta=0$.

This model is further generalized to the case where the break energy for neutrinos coming from muon decay $\left(\nu_{e}\right.$ and $\left.\nu_{\mu}\right)$ is different than that from pion decay $\left(\nu_{\mu}\right)$. Pions and muons lose energy in $p \gamma$ sources, e.g., in the presence of magnetic fields due to synchrotron losses and they may have separate break energies, $\tilde{E}_{\nu, b, \mu}$ and $\tilde{E}_{\nu, b, \pi}$. For example, for synchrotron losses, the neutrino break energy scales like $m_{i}^{5 / 2} \tau_{i}^{-1 / 2}$ for $i \in\{\pi, \mu\}$ where $m(\tau)$ is the mass (lifetime) of the particle, so the ratio of the neutrino break energies is $R_{\pi, \mu} \equiv \tilde{E}_{\nu, b, \pi} / \tilde{E}_{\nu, b, \mu} \simeq 18.4$ when synchrotron cooling dominates. The simpler BPL model introduced above is recovered when $R_{\pi, \mu}=1$. Thus, there are at most five free parameters in the BPL model: $\gamma, \Delta, \tilde{E}_{\nu, b}, R_{\pi, \mu}$, and the neutrino flux normalization $\Phi_{\nu}$.

The IceCube neutrino flux is considered to be dominantly extragalactic and compatible with a diffuse origin [9,22-24]. Hence, the expected diffuse neutrino intensity at Earth for the flavor $\nu_{\beta}(\beta=e, \mu, \tau)$ is

$$
\mathcal{I}_{\nu_{\beta}}=\sum_{\nu_{\alpha}} d_{H} \int_{0}^{z_{\max }} d z \frac{F_{\nu_{\alpha}}\left((1+z) E_{\nu}\right) \rho(z)}{h(z)} \bar{P}\left(\nu_{\alpha} \rightarrow \nu_{\beta}\right),
$$

where $d_{H}=c / H_{0}, h(z)=\sqrt{(1+z)^{3} \Omega_{m}+\Omega_{\Lambda}}$, with $\Omega_{m}=$ $0.308, \Omega_{\Lambda}=1-\Omega_{m}$, and $H_{0}=67.8 \mathrm{~km} \mathrm{~s}^{-1} \mathrm{Mpc}^{-1}$ [25]. For the redshift evolution $\rho(z)$, we assume as a benchmark case that the source luminosity density evolves as $(1+z)^{\theta}$ for $\theta=3$, up to a certain $z_{c} \simeq 1.5$, and it is constant for $z>z_{c}$ [26]. Different redshift scalings for $\theta \in[0,4]$ and $z_{c} \in[0.5,2]$ do not significantly affect our conclusions. The averaged oscillation probability is $\bar{P}\left(\nu_{\alpha} \rightarrow \nu_{\beta}\right)=$ $\sum_{i}\left|U_{\alpha i}\right|^{2}\left|U_{\beta i}\right|^{2}$, where $U$ is the standard mixing matrix $[27,28]$. For the mixing angles, we take the latest global fit results $[29,30]$. The per-flavor flux from the source, $F_{\nu_{\alpha}}$, is either a SPL or a BPL.

We then compute the corresponding per-flavor intensity expected in the two event topologies; the track intensity roughly corresponds to the $\nu_{\mu}$ one, while the cascade one corresponds to the $\nu_{e}+\nu_{\tau}$ one [31]. A scan over all possible values of each model parameter is done to compare with the IceCube neutrino data through a $\chi^{2}$ test:

$$
\chi^{2}=\sum_{i \in\{t, c\}}\left(\frac{\Phi_{i}-\Phi_{i, \mathrm{IC}}}{\sigma_{\Phi_{\nu, i}}}\right)^{2}+\left(\frac{\gamma_{i}-\gamma_{i, \mathrm{IC}}}{\sigma_{\gamma_{i}}}\right)^{2}
$$

where the sum runs on both neutrino event topologies, $\left(\Phi_{i}, \gamma_{i}\right)$ are the normalization and spectral indices at Earth, which come from our calculations, and $\left(\Phi_{i, \mathrm{IC}}, \gamma_{i, \mathrm{IC}}\right)$ fit the IceCube data. For the SPL case with two free parameters $\left(\Phi_{\nu}, \gamma\right)$, we find $\chi^{2}=13.4$, which corresponds to $3.23 \sigma$ of tension. When we expand the source model to the BPL case with four free parameters $\left(\gamma, \Delta, \tilde{E}_{\nu, b}, \Phi_{\nu}\right)$ and $R_{\pi, \mu}=1$, we find that the $\chi^{2}$ does not improve, which results in $>3.66 \sigma$ tension. That is the BPL case, which is not preferred by the data with respect to the SPL. In addition, letting $R_{\pi, \mu}$ float freely only improves the fit to $\chi^{2}=10.7$, which is disfavored at $>3.27 \sigma$ and provides only marginal improvement $(1.64 \sigma)$ over the BPL case. In this case, the best fit point has $R_{\pi, \mu}>100$ and $\Delta$ large, similar to a damped muon source.

Our findings confirm that adding a break to the source spectra provides marginal improvement to the data fit, and that a SPL fit is justified. Most importantly, the standard neutrino source scenario is disfavored at $>3.2 \sigma$ by the IceCube track and cascade data (see the left columns of Table I for a summary). While muon cooling does provide both an energy and flavor dependent effect, it is not enough to resolve the tension due to the large mixing angles. We expect that any mechanism that increases the relative number of $\nu_{\mu}$ 's at the source (such as muon damping from synchrotron cooling) at high energy will equally increase the relative number of $\nu_{\tau}$ 's after oscillations since $\theta_{23} \sim 45^{\circ}$ is minimizing the effect.

Invisible neutrino decay.-To solve the tension between the fits provided by the two event topologies, an interesting model modifying the flavor ratio in an energy dependent 
TABLE I. The $\chi^{2}$ and significance for the single power law (SPL) and broken power law (BPL) models, along with the best fit source spectral index and neutrino lifetime. Here, we fix $R_{\pi, \mu}=1$ for the BPL model, see text. The BPL models have as many or more parameters than data points; only a lower limit on the significance can be placed by taking 1 DOF.

\begin{tabular}{lccccc}
\hline \hline & \multicolumn{2}{c}{ Standard Model } & & \multicolumn{2}{c}{ Invisible $\nu$ Decay } \\
\cline { 2 - 3 } \cline { 5 - 6 } Model & SPL & BPL & & SPL & BPL \\
\hline$\chi^{2}$ & 13.4 & 13.4 & & 1.57 & 1.57 \\
$\sigma$ & 3.23 & $>3.65$ & & 1.25 & $>1.25$ \\
$\gamma$ & $2.4 \pm 0.10$ & - & & $2.73 \pm 0.10$ & - \\
$\log _{10}[(\tau / m) /(\mathrm{s} / \mathrm{eV})]$ & - & - & & $1.93_{-0.40}^{+0.26}$ & $1.93_{-0.40}^{+0.26}$ \\
\hline \hline
\end{tabular}

fashion during propagation is neutrino decay [35-38]. The latter is described by a new interaction term: $\mathcal{L} \supset g_{i j} \nu_{i} \nu_{j} \phi$, where $\phi$ is a new light $\left(m_{\phi} \lesssim m_{\nu}\right)$ or massless scalar known as the Majoron, which could provide neutrinos with their masses [39-41]. Specifically, we here focus on the invisible decay scenario where the decay products are a Majoron and a right-handed neutrino (left-handed antineutrino) [40,41]; another model of invisible neutrino decay features unparticles $[42,43]$. Depending on the mass ordering and absolute mass scale, the decay products of visible neutrino decay may have significantly less energy. For a steeply falling spectrum $(\gamma \gtrsim 2)$, visible decay becomes effectively invisible.

We assume that $\nu_{1}$ is stable since it has the least $\nu_{\mu}$ fraction, because this can suppress the $\nu_{\mu}$ fraction at low energies. This may be the case if the mass ordering is normal, as is currently favored at $2-3.4 \sigma$ [29,30,44-46], and the Majoron has a mass between $\nu_{1}$ and $\nu_{2}$, or if $\nu_{1}$ is massless (or very light) and has no (significant) coupling to the Majoron.

The oscillation averaged probability is

$$
\bar{P}\left(\nu_{\alpha} \rightarrow \nu_{\beta}\right)=\sum_{i=1}^{3}\left|U_{\alpha i}\right|^{2}\left|U_{\beta i}\right|^{2} e^{-\Lambda_{i}},
$$

where $\Lambda_{i} \equiv d_{H} f(z) m_{i} / E_{\nu} \tau_{i}$ and $f(z)=\int_{0}^{z} d z^{\prime}\left(1+z^{\prime}\right)^{-2}$ $h^{-1}\left(z^{\prime}\right)$ is the corrected cosmological distance scaling for neutrino decay [47]. We take $\Lambda_{1}=0$ and $\Lambda_{2}=\Lambda_{3} ; \tau / m$, identical for $\nu_{2}$ and $\nu_{3}$, is our free parameter.

Figure 2 shows the modification of the track vs cascade ratio due to invisible neutrino decay within the model introduced above. One can check that in order to have an effect $\left(\Lambda_{2}, \Lambda_{3} \sim 1\right)$ within the region of interest of IceCube, we should have $\tau / m \sim 10^{2} \mathrm{~s} / \mathrm{eV}$.

Minimizing the $\chi^{2}$ in the only SPL case with neutrino decay, we find $\chi^{2}=1.57$ with $\log _{10}[(\tau / m) /(\mathrm{s} / \mathrm{eV})]=$ $1.93_{-0.40}^{+0.26}$. At 1 degree of freedom (DOF), this represents a good fit, consistent with the data at $1.25 \sigma$. It is an improvement over the stable neutrino case of $\Delta \chi^{2}=11.8$,

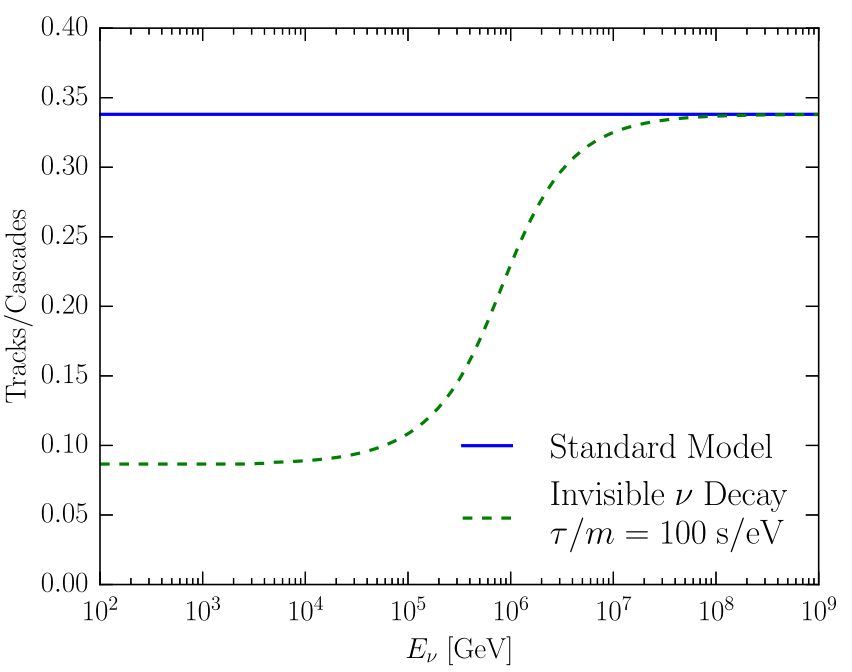

FIG. 2. The track to cascade ratio as a function of the neutrino energy. The invisible neutrino decay of $\nu_{2}$ and $\nu_{3}$ reduces the track and cascade ratio below $1 \mathrm{PeV}$ up to $75 \%$ with respect to the case where all neutrinos are stable. The deviation from the expected value of 0.5 for the standard case is mostly due to track misidentification, wherein track events are sometimes misidentified as cascades [31].

showing that the neutrino decay scenario is preferred by the data over the standard stable neutrino case by $3.4 \sigma$. The two-dimensional (2D) $\chi^{2}$ projection of the source spectral index $\gamma$ and the neutrino lifetime $\tau / m$ is shown in Fig. 3. We note that $\tau / m$ is fairly well determined since it must give observable consequences within IceCube's region of interest. Varying the redshift evolution power $\theta$ produces a fairly small effect with the best fit value of $\tau / m$, and the $\chi^{2}$ slightly changes with $\tau / m$ increasing with $\theta$. If we extend our fit to the BPL source model, the best fit point does not change at all and $\Delta=0$ is preferred, see Table I [48].

Our findings should be compared with existing bounds on invisible neutrino decay. The best terrestrial constraints on invisible $\nu_{3}$ decay come from atmospheric and longbaseline data: $\log _{10}\left[\left(\tau_{3} / m_{3}\right) /(\mathrm{s} / \mathrm{eV})\right]>-9.52 \quad[51,52]$; the best terrestrial constraints on invisible $\nu_{2}$ decay are from solar neutrinos and are $\log _{10}\left[\left(\tau_{2} / m_{2}\right) /(\mathrm{s} / \mathrm{eV})\right]>$ -3.15 [53,54]. Hints for $\nu_{3}$ invisible decay exist at $\log _{10}\left[\left(\tau_{3} / m_{3}\right) /(\mathrm{s} / \mathrm{eV})\right] \sim-11[55,56]$.

Strong constraints, in apparent contradiction with our findings, have been derived from SN 1987A: $\log _{10}[(\tau / m) /(\mathrm{s} / \mathrm{eV})] \gtrsim 5$ [57]; however, these constraints only apply to $\bar{\nu}_{e}$ measurements under the assumption that all neutrino mass eigenstates are decaying and should be considered with caution. Even in the case of full $\nu_{2}$ and $\nu_{3}$ decay, the $\bar{\nu}_{e} \rightarrow \bar{\nu}_{e}$ oscillation averaged probability would be suppressed by $16 \%$, which is still smaller than the SN 1987A statistical uncertainties ( 20\%) and current theoretical uncertainties. IceCube data have been used to place a constraint on the neutrino lifetime at $\log _{10}[(\tau / m) /(\mathrm{s} / \mathrm{eV})] \gtrsim 1$ by assuming that neutrinos do 


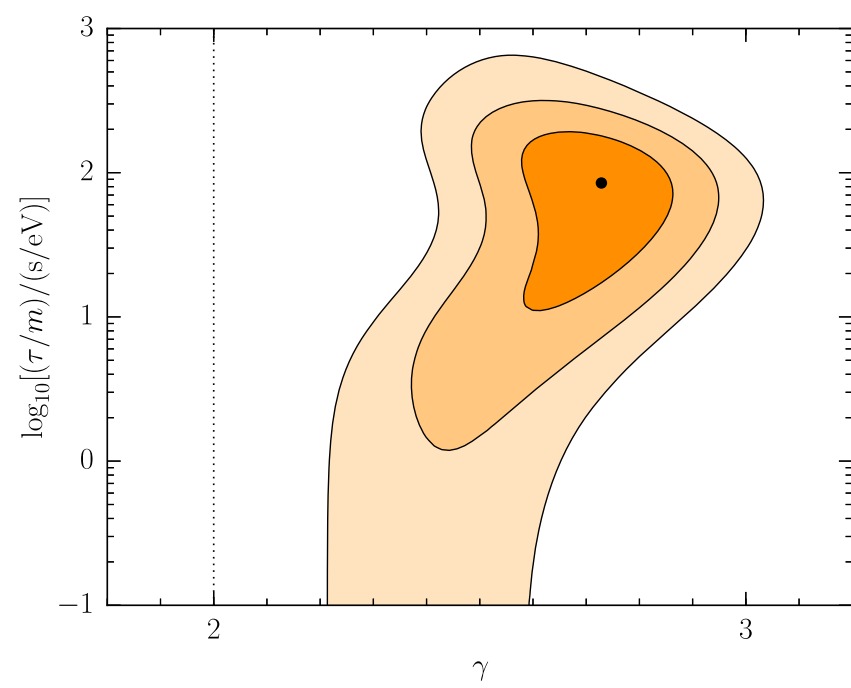

FIG. 3. The 2D $\chi^{2}$ projection for neutrino decay with a single power law astrophysical flux. The shaded regions represent $1,2,3 \sigma$ for 2 DOF. The best fit point of $\gamma=2.73$ and $\log _{10}[(\tau / m) /(\mathrm{s} / \mathrm{eV})]=1.93$, indicated with the dot, has $\chi^{2}=1.57$. This includes a marginalization over the source normalization. The slight preference for the full decay case over the $\nu \mathrm{SM}$ is because it modifies the relative normalization of the track and cascade diffuse intensities.

not fully decay within the IceCube energy range [58,59], which is not the case considered here. The most stringent constraints on the lifetime of neutrinos have been derived from cosmic microwave background data at the level of $\log _{10}[(\tau / m) /(\mathrm{s} / \mathrm{eV})] \gtrsim 11[60]$. Noticeably, these bounds can be alleviated in the event that only one or two neutrinos decay, and the remaining ones are free streaming [61-63] and are therefore not in contradiction with our findings. Interestingly, neutrino decay, with parameters similar to our model, was proposed as an alternate solution to the solar neutrino problem [64].

Other possible interpretations. - Another possible explanation of the tension between the track and cascade data sets is the decay of dark matter (DM) [65] to electron neutrinos $\left(\chi \rightarrow \nu_{e} \bar{\nu}_{e}\right)$. We focus on DM decay instead of annihilation, as the galactic anisotropy constraints $[22,23,66]$ are weaker for DM decay since the DM annihilation peaked more towards the Galactic center. In order to estimate the expected track and cascade distribution, the galactic and extragalactic diffuse intensity of neutrinos is computed, including electroweak corrections, by using PYTHIA 8.2 [67] and a Navarro-Frenk-White galactic DM profile [68].

While a good quality of fit $\left(\chi^{2}<1\right)$ is found in a SPL + DM model with four parameters $\left(\tau_{\chi} \mathrm{s}, m_{\chi} \mathrm{TeV}, \Phi_{\nu}, \gamma\right)$, this model has a number of undesirable properties. The galactic contribution to the flux peaks at energies below the cascade flux sensitivity, and its contributions, due to the typical energy uncertainty of cascades, are $\gtrsim 15 \%$ [69]; this results in a contribution to the cascade flux at low energies due to the energy uncertainty, but a minimal contribution to the track flux (after oscillations). The resultant peak flux is larger than the measured flux at energies just below the region of interest for IceCube's cascade analysis. From $\mathrm{SU}(2)$ symmetry, there will be an $e^{+} e^{-}$channel, leading to $\gamma$-rays from electroweak corrections constrained by the Fermi large area telescope [70]. Finally, this fit requires a short DM lifetime, which is strongly constrained by the cosmic microwave background and bounds from the reionization epoch, the best fit values being $\tau_{\chi} \sim 10^{23} \mathrm{~s}$, $m_{\chi} \sim 10 \mathrm{TeV}[71,72]$. All considered, DM decay does not seem to resolve this tension.

Several additional effects could provide an energy and flavor dependent modification of the standard neutrino flux from an astrophysical source. For example, the Glashow resonance occurs when a $\bar{\nu}_{e}$ with $E_{\nu}=6.3 \mathrm{PeV}$ scatters off an electron in the ice, creating an on shell $W^{-}$[73], increasing IceCube's sensitivity in that energy range considerably. IceCube performs their fits assuming that $\mathcal{I}_{\nu}=\mathcal{I}_{\bar{\nu}}$. While this is generally the case if neutrinos are mainly produced through $p p$ interactions, it will not be the case if the main neutrino production channel is $p \gamma$ interactions [74]. For the SPL case, $\mathcal{I}_{\nu_{e}} / \mathcal{I}_{\bar{\nu}_{e}} \simeq 3.5$, which would somewhat harden the cascade spectrum but would not be enough to reduce the tension of the fit.

Another option that could alleviate the track vs cascade fit tension is neutron decay sources. Neutrons decay to $\bar{\nu}_{e}$ 's, and they are produced alongside charged pions in $p \gamma$ interactions (as well as in $p p$ interactions); thus, they are expected to provide an additional contribution of $\nu_{e}$ 's to the high energy astrophysical neutrino flux. The energy of neutrinos from neutrons is suppressed by about two orders of magnitude compared with those from pion decay. However, for a spectral index $\gtrsim 2$ as in our case, this contribution is subleading. Neutrons also result from the photodisintegration of heavy ions in dense sources, although this flux is also suppressed compared to the standard contribution by at least an order of magnitude [75,76].

In addition, nonstandard neutrino interactions with ultralight mediators $\left(m_{Z^{\prime}} \ll 1 \mathrm{eV}\right)$, as well as pseudo-Dirac neutrino models [77,78], may also affect the track vs cascade ratio. However, in both cases, we expect an impact on the neutrino data set that is smaller than the one induced by the invisible neutrino decay scenario.

A solution to the $\nu_{\tau}$ observational deficit.-The IceCube detector is expected to observe 2 or $3 \nu_{\tau}$ events in the energy range of interest $[49,79]$. However, currently no $\nu_{\tau}$ events are observed. The assumption of invisible neutrino decay for the $\nu_{2}$ and $\nu_{3}$ eigenstates would induce a reduction of $\mathcal{I}_{\nu_{\tau}}$ of $80 \%$ below $1 \mathrm{PeV}$, which, convolved with the detection efficiency, leads to a $\sim 59 \%$ reduction in the number of $\nu_{\tau}$ events for our best fit value $\tau / m=$ $10^{2} \mathrm{~s} / \mathrm{eV}$. The invisible neutrino decay could then also explain the current deficit of $\nu_{\tau}$ events. 
Conclusions.-The IceCube Observatory detects high energy astrophysical neutrinos through two event topologies: tracks and cascades. By simultaneously taking advantage of the energy and flavor information present in the two data sets, for the first time we have placed strong constraints on the consistency of the data with the standard source picture. A conventional model for the neutrino production in astrophysical sources is unable to simultaneously explain the track and cascade data at $>3 \sigma$.

We tested several new physics models and found that the invisible neutrino decay of $\nu_{2}$ and $\nu_{3}$ with $\tau / m=10^{2} \mathrm{~s} / \mathrm{eV}$ is preferred by the IceCube data by $3.4 \sigma$, and it is consistent with all other existing constraints. While this model is more natural in the normal mass ordering, it is consistent with either ordering. In addition, a model of visible decay in $\nu_{1}$ may provide additional improvements to the fit by producing additional $\nu_{1}$ 's (mostly $\nu_{e}$ 's) at lower energies. Interestingly, our model also predicts a 59\% reduction in the number of expected $\nu_{\tau}$ events reconciling the current observational deficit.

As more neutrino data arrives with the advent of IceCube-Gen2 [80] and KM3NeT [81], and the spectral distributions will be defined more precisely for both event topologies, it will be possible to further test our result.

We are grateful to Mauricio Bustamante, Steen Hannestad, Rebecca Leane, Orlando Peres, and Mohamed Rameez for useful discussions. P. B. D. and I. T. acknowledge support from the Villum Foundation (Project No. 13164) and the Danish National Research Foundation (DNRF91). P. B. D. thanks the Danish National Research Foundation (Grant No. 1041811001) for support. The work of I. T. has also been supported by the Knud Højgaard Foundation and the Deutsche Forschungsgemeinschaft through Sonderforschungbereich SFB 1258 "Neutrinos and Dark Matter in Astro- and Particle Physics" (NDM).

*denton@nbi.ku.dk tamborra@nbi.ku.dk

[1] M. G. Aartsen et al. (IceCube Collaboration), Phys. Rev. Lett. 111, 021103 (2013).

[2] M. G. Aartsen et al. (IceCube Collaboration), Science 342, 1242856 (2013).

[3] M. G. Aartsen et al. (IceCube Collaboration), Astrophys. J. 833, 3 (2016).

[4] L. A. Anchordoqui et al., JHEAp 1-2, 1 (2014).

[5] P. Mészáros, arXiv:1511.01396.

[6] E. Waxman, arXiv:1511.00815.

[7] K. Murase, arXiv:1511.01590.

[8] Y. Farzan and A. Y. Smirnov, Nucl. Phys. B805, 356 (2008).

[9] M. G. Aartsen et al. (IceCube Collaboration), Astrophys. J. 809, 98 (2015).

[10] C.-Y. Chen, P. S. B. Dev, and A. Soni, Phys. Rev. D 92, 073001 (2015).

[11] A. Palladino and F. Vissani, Astrophys. J. 826, 185 (2016).
[12] L. A. Anchordoqui, M. M. Block, L. Durand, P. Ha, J. F. Soriano, and T. J. Weiler, Phys. Rev. D 95, 083009 (2017).

[13] A. Palladino and W. Winter, Astron. Astrophys. 615, A168 (2018).

[14] M. Chianese, R. Mele, G. Miele, P. Migliozzi, and S. Morisi, Astrophys. J. 851, 36 (2017).

[15] A. C. Vincent, S. Palomares-Ruiz, and O. Mena, Phys. Rev. D 94, 023009 (2016).

[16] S. Palomares-Ruiz, A. C. Vincent, and O. Mena, Phys. Rev. D 91, 103008 (2015).

[17] Y. Sui and P. S. Bhupal Dev, J. Cosmol. Astropart. Phys. 07 (2018) 020.

[18] P. B. Denton and I. Tamborra, J. Cosmol. Astropart. Phys. 04 (2018) 058.

[19] H. M. Niederhausen, M. Lesiak-Bzdak, and A. Stoessl (IceCube Collaboration), Proceedings, 34th International Cosmic Ray Conference (ICRC 2015): The Hague, The Netherlands, 2015, Proc. Sci., ICRC2015, (2016) 1109.

[20] Neutrino oscillations already provide evidence of physics beyond the Standard Model in that they have mass. In this Letter, New Physics refers to physics beyond both the Standard Model and the fact that neutrinos have mass.

[21] P. Mészáros, Annu. Rev. Astron. Astrophys. 40, 137 (2002).

[22] P. B. Denton, D. Marfatia, and T. J. Weiler, J. Cosmol. Astropart. Phys. 08 (2017) 033.

[23] M. G. Aartsen et al. (IceCube Collaboration), Astrophys. J. 849, 67 (2017).

[24] S. Ando, I. Tamborra, and F. Zandanel, Phys. Rev. Lett. 115, 221101 (2015).

[25] P. A. R. Ade et al. (Planck Collaboration), Astron. Astrophys. 594, A13 (2016).

[26] C. Gruppioni et al., Mon. Not. R. Astron. Soc. 432, 23 (2013).

[27] Z. Maki, M. Nakagawa, and S. Sakata, Prog. Theor. Phys. 28, 870 (1962).

[28] B. Pontecorvo, Zh. Eksp. Teor. Fiz. 53, 1717 (1967); [Sov. Phys. JETP 26, 984 (1968)].

[29] I. Esteban, M. C. Gonzalez-Garcia, M. Maltoni, I. MartinezSoler, and T. Schwetz, J. High Energy Phys. 01 (2017) 087.

[30] "Nufit 3.2," nu-fit.org (2018).

[31] See Supplemental Material at http://link.aps.org/ supplemental/10.1103/PhysRevLett.121.121802 for technical details, which includes Refs. [32-34].

[32] M. G. Aartsen et al. (IceCube Collaboration), Phys. Rev. Lett. 114, 171102 (2015).

[33] R. Gandhi, C. Quigg, M. H. Reno, and I. Sarcevic, Astropart. Phys. 5, 81 (1996).

[34] C. Patrignani et al. (Particle Data Group), Chin. Phys. C 40, 100001 (2016).

[35] J. F. Beacom, N. F. Bell, D. Hooper, S. Pakvasa, and T. J. Weiler, Phys. Rev. Lett. 90, 181301 (2003).

[36] I. M. Shoemaker and K. Murase, Phys. Rev. D 93, 085004 (2016).

[37] Z. Moss, M. H. Moulai, C. A. Argüelles, and J. M. Conrad, Phys. Rev. D 97, 055017 (2018).

[38] S. Choubey, S. Goswami, and D. Pramanik, J. High Energy Phys. 02 (2018) 055.

[39] A. Acker, S. Pakvasa, and J. T. Pantaleone, Phys. Rev. D 45, R1 (1992).

[40] G. B. Gelmini and M. Roncadelli, Phys. Lett. 99B, 411 (1981). 
[41] Y. Chikashige, R. N. Mohapatra, and R. D. Peccei, Phys. Lett. 98B, 265 (1981).

[42] H. Georgi, Phys. Rev. Lett. 98, 221601 (2007).

[43] S. Zhou, Phys. Lett. B 659, 336 (2008).

[44] P. F. de Salas, D. V. Forero, C. A. Ternes, M. Tortola, and J. W. F. Valle, Phys. Lett. B 782, 633 (2018).

[45] Valencia-Globalfit, globalfit.astroparticles.es (2018).

[46] F. Capozzi, E. Lisi, A. Marrone, and A. Palazzo, Prog. Part. Nucl. Phys. 102, 48 (2018).

[47] P. Baerwald, M. Bustamante, and W. Winter, J. Cosmol. Astropart. Phys. 10 (2012) 020.

[48] A newer unpublished analysis from the IceCube Collaboration [49] slightly changes the various qualities of fit related to the track and cascade data sets. Given the different energy ranges, the tension between the track and cascade data decreases to $2.5 \sigma$ for the SPL. However, we find that this does not significantly change our conclusions and neutrino decay is still preferred at $2.8 \sigma$. This trend has also been confirmed from the preliminary results presented at Neutrino 2018 [50].

[49] M. G. Aartsen et al. (IceCube Collaboration Collaboration), arXiv:1710.01191.

[50] I. Taboada, A View of the Universe with the IceCube and ANTARES Neutrino Telescopes (2018), https://doi.org/10 .5281/zenodo.1286918.

[51] M. C. Gonzalez-Garcia and M. Maltoni, Phys. Lett. B 663, 405 (2008).

[52] G. Pagliaroli, N. D. Marco, and M. Mannarelli, Phys. Rev. D 93, 113011 (2016).

[53] J. M. Berryman, A. de Gouvea, and D. Hernandez, Phys. Rev. D 92, 073003 (2015).

[54] R. Picoreti, M. M. Guzzo, P. C. de Holanda, and O. L. G. Peres, Phys. Lett. B 761, 70 (2016).

[55] R. A. Gomes, A. L. G. Gomes, and O. L. G. Peres, Phys. Lett. B 740, 345 (2015).

[56] S. Choubey, D. Dutta, and D. Pramanik, J. High Energy Phys. 08 (2018) 141.

[57] K. Hirata et al., Phys. Rev. Lett. 58, 1490 (1987).

[58] G. Pagliaroli, A. Palladino, F. L. Villante, and F. Vissani, Phys. Rev. D 92, 113008 (2015).

[59] M. Bustamante, J. F. Beacom, and K. Murase, Phys. Rev. D 95, 063013 (2017).

[60] S. Hannestad and G. G. Raffelt, Phys. Rev. D 72, 103514 (2005).
[61] N. F. Bell, E. Pierpaoli, and K. Sigurdson, Phys. Rev. D 73, 063523 (2006).

[62] M. Archidiacono, S. Hannestad, R. S. Hansen, and T. Tram, Phys. Rev. D 91, 065021 (2015).

[63] S. Gariazzo, C. Giunti, and M. Laveder, arXiv:1404.6160.

[64] J. N. Bahcall, S. T. Petcov, S. Toshev, and J. W. F. Valle, Phys. Lett. B 181, 369 (1986).

[65] M. G. Aartsen et al. (IceCube Collaboration Collaboration), arXiv:1804.03848.

[66] M. Ahlers, Y. Bai, V. Barger, and R. Lu, Phys. Rev. D 93, 013009 (2016).

[67] T. Sjöstrand, S. Ask, J. R. Christiansen, R. Corke, N. Desai, P. Ilten, S. Mrenna, S. Prestel, C. O. Rasmussen, and P. Z. Skands, Comput. Phys. Commun. 191, 159 (2015).

[68] J. F. Navarro, C. S. Frenk, and S. D. M. White, Astrophys. J. 462, 563 (1996).

[69] M. G. Aartsen et al. (IceCube Collaboration Collaboration), in Proceedings, 34th International Cosmic Ray Conference (ICRC 2015): The Hague, The Netherlands, 2015 (2015), http://pos.sissa.it/cgi-bin/reader/conf.cgi? confid=236.

[70] K. Murase, R. Laha, S. Ando, and M. Ahlers, Phys. Rev. Lett. 115, 071301 (2015).

[71] H. Liu, T. R. Slatyer, and J. Zavala, Phys. Rev. D 94, 063507 (2016).

[72] T. R. Slatyer and C.-L. Wu, Phys. Rev. D 95, 023010 (2017).

[73] S. L. Glashow, Phys. Rev. 118, 316 (1960).

[74] H. Nunokawa, B. Panes, and R. Zukanovich Funchal, J. Cosmol. Astropart. Phys. 10 (2016) 036.

[75] D. Biehl, D. Boncioli, A. Fedynitch, and W. Winter, Astron. Astrophys. 611, A101 (2018).

[76] X. Rodrigues, A. Fedynitch, S. Gao, D. Boncioli, and W. Winter, Astrophys. J. 854, 54 (2018).

[77] L. Wolfenstein, Nucl. Phys. B186, 147 (1981).

[78] S. Pakvasa, A. Joshipura, and S. Mohanty, Phys. Rev. Lett. 110, 171802 (2013).

[79] A. Palladino, C. Mascaretti, and F. Vissani, J. Cosmol. Astropart. Phys. 08 (2018) 004.

[80] M. G. Aartsen et al. (IceCube Collaboration), Proceedings, Frontier Research in Astrophysics-II: Mondello, Palermo, Italy, May 23-28, 2016, Proc. Sci., FRAPWS2016, 004 (2017).

[81] S. Adrian-Martinez et al. (KM3NeT), J. Phys. G 43, 084001 (2016). 